\title{
ARTÍCULOS
}

\section{ITINERARIOS DE LA EDUCACIÓN PRIVADA EN PERÚ*}

\author{
Ricardo Cuenca ${ }^{1}$ \\ LuCiana Reátegui Amat y LeÓN ${ }^{1}$ (D) \\ SARITA ORÉ ${ }^{2}$
}

RESUMEN: La educación privada en Perú inició su periodo de mayor auge en la segunda mitad de la década de los años noventa. Específicamente, fue en 1996 cuando en el segundo gobierno de Alberto Fujimori (1995-2000) se promovió la liberalización del mercado educativo. A partir de esa fecha, tanto la oferta del servicio educativo, como la matrícula privada crecen de manera sostenida y, luego de 20 ańos, de manera aparentemente irreversible, particularmente en las zonas urbanas. El objetivo de este artículo es mostrar el itinerario que ha seguido la educación privada en Perú, a partir de la explicación del crecimiento de las matrículas en las ciudades. Para ello se ha estudiado el comportamiento de tres variables: el incremento de la oferta educativa, el crecimiento económico de las familias y la disminución del tamaño de las familias. De manera general, los resultados evidenciaron que la matrícula escolar crece debido a crecimiento económico de las familias, el incremento de la oferta privada y la disminución en el tamaño familia.

Palabras clave: Educación privada. Matrícula escolar. Educación urbana. Perú.

\section{ITINERÁRIOS DA EDUCAÇão PRIVADA NO PERU}

RESUMO: A educação privada no Peru começou seu período de maior crescimento na segunda metade da década de 1990, especificamente em 1996, quando o segundo governo de Alberto Fujimori (1995-2000) promoveu a liberalizaçáo do mercado educacional. A partir dessa data, tanto a oferta de serviços educacionais quanto a inscriçáo privada aumentaram de forma constante e, após 20 anos, parecem irreversíveis, particularmente em áreas urbanas. $\mathrm{O}$ objetivo deste artigo é mostrar o itinerário seguido pelo ensino privado no Peru, com base na explicação do crescimento das matrículas nas cidades. Para isso, estudou-se o comportamento de três variáveis: aumento da oferta educacional, crescimento econômico das famílias e diminuição do tamanho das famílias. Em geral, os resultados evidenciaram que a matrícula escolar cresceu em razão do crescimento econômico das famílias, do incremento da oferta privada e da diminuição do tamanho da família.

Palavras-chave: Educação privada. Matrícula escolar. Educação urbana. Peru.

\footnotetext{
*Este artículo es parte del proyecto de investigación "Trayectorias Educativas, Juventud y Desigualdades Sociales", financiado por la Fundación Ford.

${ }^{1}$ Instituto de Estudios Peruanos - Lima, Perú. E-mails: rcuenca@iep.org.pe; Ireategui@iep.org.pe

${ }^{2}$ Universidad de Los Andes - Bogotá, Colombia. E-mail: sp.ore@uniandes.edu.co

DOI: 10.1590/ES0101-73302019192469
} 


\title{
ITINERARIES OF PRIVATE EDUCATION IN PERU
}

\begin{abstract}
Private education in Peru began its period of greatest boom in the second half of the decade of the 1990s. Specifically, it was in 1996 when the second government of Alberto Fujimori (1995-2000) promoted the liberalization of the educational market. As of that date, both the educational service offers and the private enrollment increase steadily and, after 20 years, it was apparently irreversible, particularly in urban areas. The objective of this article is to show the itinerary followed by private education in Peru, based on the explanation of the growth of enrollment in cities. For this, the behavior of three variables has been studied: the increase of the educational offer, the economic growth of the families and the decrease in the size of the families. In general, the results showed that school enrolment grows due to the economic growth of families, the increase in private supply and the decrease in family size.
\end{abstract}

Keywords: Private education. School enrollment. Urban education. Peru.

\section{Introducción}

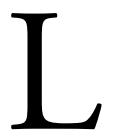

a educación privada en Perú inició su periodo de mayor auge a partir de la segunda mitad de la década de los años noventa. Específicamente, fue en 1996 cuando en el segundo gobierno de Alberto Fujimori (1995-2000) se promovió la liberalización del mercado educativo, a través de la promulgación de un decreto legislativo (DL 882) que impulsó la inversión privada por medio de beneficios tributarios y con bajos niveles de regulación estatal. A partir de esa fecha, tanto la oferta del servicio educativo, como la matrícula privada crecen de manera sostenida y, luego de 20 ańos, de manera aparentemente irreversible, particularmente en las zonas urbanas. Aun cuando a nivel nacional el porcentaje de escuelas privadas es del 25,5\%, en Lima Metropolitana, que concentra el 30\% de la matriculada nacional, la matricula privada representa el 48,7\%, como se puede apreciar en la Tabla 1.

Cabe mencionar que el sistema educativo peruano se organiza en etapas, niveles, modalidades, ciclos y programas. La Educación Básica es la primera etapa del sistema, es obligatoria y cuando la imparte el Estado es gratuita. Esta, tiene una duración de doce años y cuenta con tres niveles: inicial (un año), primaria (seis años) y secundaria (cinco años). Si bien la educación básica privada se organiza del mismo modo, esta puede ser financiada por agentes particulares como por ejemplo padres de familia, iglesias o empresas (GUADALUPE et al., 2017).

El objetivo de este artículo es mostrar el itinerario que ha seguido la educación privada en Perú. Esto desde la explicación del crecimiento de las matrí- 
culas en las ciudades y a partir del comportamiento de tres variables: el incremento de la oferta educativa, el crecimiento económico de las familias y la disminución del tamaño de las familias.

Nuestra hipótesis principal de trabajo es que la demanda por servicios privados de educación básica se expandiría en un escenario donde el incremento de la oferta privada aumenta, el crecimiento económico de las familias, al menos se sostiene, y el tamaño de las familias se mantiene o, en todo caso, disminuye.

La expansión de la educación privada en Perú es un asunto fundamental de investigación; particularmente, en un contexto donde se evidencian marcadas desigualdades educativas (BELLO \& VILLARÁN, 2004; BENAVIDES, 2007; BENAVIDES et al., 2015; CASTRO \& YAMADA, 2011; CUENCA \& PATIÑO, 2014; CUENCA \& REÁTEGUI, 2016; DE BELAUNDE, 2011). En ese sentido, resulta importante ofrecer información sobre las consecuencias de un mercado educativo privado, muy heterogéneo y altamente desregulado, en la alta segregación educativa que existe en el país (BENAVIDES; LEÓN; ETESSE, 2014; MURILLO, 2016; RIVAS, 2015).

En Perú, de manera general, los estudios se han concentrado en comprender las razones por las que las familias optan por la educación privada, en línea con los enfoques de school choice (BAGLEY; WOODS; GLATTER, 2005). Nuestra orientación es, por el contrario, hacia los factores de corte estructural (demográficos, económicos, políticos) que podrían explicar el crecimiento de la

\section{Tabla 1}

Evolución de la tasa de matrícula de educación básica regular por gestión.

\begin{tabular}{l|c|c|c|c}
\hline \multirow{2}{*}{ Año } & \multicolumn{2}{|c|}{ Nacional } & \multicolumn{2}{c}{ Lima } \\
\cline { 2 - 5 } & Estatal (\%) & Privada (\%) & Estatal (\%) & Privada (\%) \\
\hline 2008 & 80,0 & 20,0 & 59,7 & 40,3 \\
\hline 2009 & 79,0 & 21,0 & 57,9 & 42,1 \\
\hline 2010 & 78,4 & 21,6 & 56,4 & 43,6 \\
\hline 2011 & 77,1 & 22,9 & 54,8 & 45,2 \\
\hline 2012 & 75,8 & 24,2 & 53,0 & 47,0 \\
\hline 2013 & 74,4 & 25,6 & 50,7 & 49,3 \\
\hline 2014 & 73,9 & 26,1 & 49,7 & 50,3 \\
\hline 2015 & 74,0 & 26,0 & 49,8 & 50,2 \\
\hline 2016 & 74,3 & 25,7 & 50,7 & 49,3 \\
\hline 2017 & 74,9 & 25,1 & 51,7 & 48,3 \\
\hline 2018 & 74,5 & 25,5 & 51,3 & 48,7 \\
\hline
\end{tabular}

Fuente: Censos Escolares, Unidad de Estadística Educativa, Ministerio de Educación. 
matrícula privada en la educación escolar. Sin duda, constituyen aproximaciones complementarias para entender este complejo problema de estudio.

\section{Revisión de literatura}

Los estudios sobre la oferta de la educación privada en Perú son diversos. $\mathrm{Si}$ bien existe algunas investigaciones relacionadas al nivel superior de enseñanza (BENAVIDES et al., 2015; CUENCA \& REÁTEGUI, 2016; LAMAS, 2015), la gran mayoría de estos se han concentrado en la educación básica. Este último grupo de estudios se puede dividir en dos grandes periodos. Por un lado, se encuentran los elaborados entre el año 1996 y el 2004, que buscaron mostrar fundamentalmente las ventajas de la educación privada en un contexto de liberalización del mercado educativo. Y, por el otro, se encuentran los elaborados a partir del 2011 que buscaron comprender el proceso de expansión de la escuela privada.

Durante el primer periodo, caracterizado por la "defensa de la educación privada”, pertenece el informe producido por Díaz et al. (1995) que sostenía que los mecanismos reguladores del Ministerio de Educación (MINEDU) no permitían la expansión (necesaria) de la educación privada en Lima. También están los trabajos de Ortiz de Zevallos et al. (1999) sobre las reformas institucionales que se produjeron en los noventa y el de Du Bois, Chávez y Cusanto (2004) que realiza un diagnóstico sobre las políticas sociales durante el mismo periodo.

En el caso de Du Bois, Chávez y Cusanto (2004), el financiamiento de la educación privada se presenta como necesario, ya que permite que las familias puedan optar por el tipo de educación que quieren para sus hijos, dentro de un esquema de competencia. En esta línea, el subsidio del Estado a la educación privada se presenta como una de las opciones más viables para atender el problema educativo en Perú (ORTIZ DE ZEVALLOS et al., 1999). En ese sentido, para los autores, la promoción de la oferta privada de parte del Estado se constituye como una de las principales vías para mejorar la calidad de la educación.

El segundo grupo de estudios, como se ha señalado, se inicia en el año 2011. A diferencia de los reportes correspondientes al primer periodo, en este lapso los informes buscaron comprender el proceso de crecimiento de la educación privada en Perú, a partir de las características de la oferta y de la comprensión del tipo de demanda. Con relación a la oferta, los estudios señalan que existe un marco legal que continúa favoreciendo la apertura y expansión de las escuelas privadas (BALARÍN et al., 2018; BALARÍN, 2016; CUENCA, 2013).

En Perú, la oferta educativa privada es bastante heterogénea y se ha caracterizado por la aparición de colegios de carácter corporativo, que buscan presentarse como escuelas de calidad (ROMÁN \& RAMÍREZ, 2018) y por la coexistencia de instituciones privadas de muy bajo y muy alto costo (BALARÍN 
et al., 2018; CUENCA, 2013). No obstante, a pesar de la heterogeneidad en la oferta, la composición social del alumnado al interior de estos colegios es bastante homogénea (BALARÍN, 2016; MIRANDA, 2008).

Respecto a la demanda, Sanz (2014) indica que la preferencia por una opción privada de parte de las familias se debe a una idea de progreso y movilidad social a través de la educación. Es decir, la educación privada es vista como una oportunidad para obtener estatus y diferenciarse socialmente. En esta línea, Sparrow y Ponce de León (2015) indican que existe una creencia generalizada de que la educación privada es de mejor calidad, no solo por el desempeño de los docentes, sino también porque al pagar una mensualidad sienten que tienen más derecho de exigir un mejor servicio. Independientemente de los resultados, el desplazamiento de la matrícula escolar hacia la oferta privada crece debido a la instalación de un imaginario social que Cuenca (2013) llama de "calidad per se" o por la falta de referentes sobre calidad educativa que tienen las familias, tal como lo sostienen Balarín et al. (2018). Las escuelas privadas de bajo y alto costo responden también a las posibilidades económicas que tienen las familias que la componen, lo que se traduce en ventajas comparativas (ej. infraestructura) de una respecto a la otra (BALARÍN et al., 2018). Así, las escuelas privadas ubicadas, por ejemplo, en los barrios de nivel socioeconómico más alto de Lima son las que obtienen mejores resultados en relación con las escuelas privadas ubicadas en sectores más bajos; en contraparte, las escuelas públicas ubicadas en barrios más pobres las que obtienen mejores resultados frente a las privadas de estas mismas zonas (CUENCA, 2013), según las evaluaciones estandarizadas de estudiantes, realizadas anualmente por el Ministerio de Educación.

Balarín (2016), Román y Ramírez (2018) y Sanz (2015) coinciden en que las decisiones son un proceso complejo en las familias. Aun cuando permanecen cierta presencia de una ideología individualista y emprendedora, así como estrategias para la mejora de posiciones sociales, hay asuntos de orden práctico y de "bienestar de los niños" (BAGLEY; WOODS; GLATTER, 2005) presentes de manera evidente en las opciones de las familias. Esto está asociado a una idea de calidad utilitarista de la educación; es decir, a la concentración de éxito vinculado al aprendizaje de ciertas áreas curriculares (como matemática y lectura); es decir, el desempeño y la productividad (CUENCA, 2012); reflejada, por ejemplo, en cómo las familias valoran calidad educativa como la promesa de las escuelas privadas que "aseguran" mayores posibilidades de seguir estudios de educación superior (BALARÍN et al., 2018).

En esta línea, si bien se mantiene la idea de la educación como vehículo de movilidad social, es a través de la educación privada que "se convierte en un proyecto de superación personal, espíritu emprendedor y acceso a lo privado, a no depender del Estado" (ROMÁN \& RAMÍREZ, 2018, p. 57). Con relación a esto, 
Sanz (2015) señala que esta situación produce prácticas de diferencia social, ya que el ingreso a las escuelas públicas constituye un "fracaso" dentro de las trayectorias familiares. Esto debido a que constituye un referente opuesto a la trayectoria ascendente a la que se pretende llegar.

En menor medida, tenemos informes que presentan la efectividad en los aprendizajes en función al tipo de oferta, en donde se confirmar que las condiciones socioeconómicas de las familias y el entorno tienen un peso determinante en el logro académico de los estudiantes, antes que el tipo de escuela (BENAVIDES; LEÓN; ETESSE, 2014; MIRANDA, 2008).

Algunos estudios dan cuenta que en las decisiones de las familias los temas asociados a la "calidad" no son desencadenantes. En efecto, según el último reporte del 2017 de la Oficina de Medición de la Calidad del Ministerio de Educación, los resultados de las pruebas de comprensión lectora y matemática de las escuelas públicas han superado a los resultados de las escuelas privadas. Recientes estudios sobre la efectividad de la educación privada identifican que no es posible establecer una correlación directa entre el tipo de gestión y el logro de aprendizajes. Los informes colombianos Iregui, Melo y Ramos (2007) y de Núnez et al. (2002), así como los estudios producidos en Chile por Bellei (2007) y Larrañaga (2004) y las reflexiones hechas por Robertson et al. (2012) sobre la educación en Estados Unidos, coinciden en que la oferta privada no es más efectiva que la pública, y que los logros exhibidos son atribuibles más a condiciones económicas que a variables propiamente educativas.

Finalmente, resulta difícil establecer una clasificación sobre el proceso de privatización educativa en Perú, pues no existen políticas de subsidios que mostraría la privatización "directa" de la educación estatal. Sin embargo, tal como lo sostiene Balarín (2015; 2016), las características de cómo se desarrolló el crecimiento de la demanda por educación privada podrían ser entendidas bajo el modelo de privatización por defecto. Tomando la categoría desarrollada por Caddell y Ashley (2006), Balarín indica que, en Perú, "Lo que encontramos, entonces, no es un proceso de privatización de la educación pública mediante la introducción de mecanismos de mercado, sino más bien un proceso de privatización por defecto" (BALARÍN, 2016, p. 187); en el que las presiones de las familias, en un contexto de crecimiento económico y descuido del estado, han favorecido el crecimiento de la oferta privada.

Aun cuando coincidimos con la afirmación, sostenemos que la privatización educativa en Perú es una consecuencia de la promoción del Estado, en un contexto de instalación de políticas neoliberales tales como la liberalización del mercado, la ausencia de regulación, los estímulos a la inversión privada y el desprestigio de "lo público" (CUENCA, 2016). 


\section{Metodología}

Buscamos mostrar la relación en las principales ciudades entre el crecimiento de la matrícula privada y el crecimiento del tamańo familiar, del ingreso per cápita de los hogares, y de la oferta de escuelas privadas. El periodo que se analiza es del 2004 al 2016, al ser el periodo para el cual existe información completa en las variables de interés.

Tanto la variable de tamańo familiar como el gasto per cápita de los hogares son construidas a partir de la Encuesta Nacional de Hogares (ENAHO) del Instituto Nacional de Estadística e Informática (INEI); la variable de oferta de escuelas privadas proviene del Censo Escolar del Ministerio de Educación (MINEDU). Se calcula la tasa de crecimiento de las variables entre los ańos 2004 y 2016. Dado que las tasas de crecimiento se ven afectadas por el tamaño inicial (ciudades pequeńas presentan crecimientos más grandes que ciudades grandes), se optó por clasificar las ciudades según tamaños.

De acuerdo con el INEI (2017), es de 75 el número de ciudades principales en Perú, las cuales albergan el 62,8\% de la población nacional. Las 74 ciudades para las que existe información completa fueron clasificadas en cuatro grupos de acuerdo con su tamaño poblacional del año 2007:

- ciudades pequeñas, de menos de 100.000 habitantes;

- ciudades medianas, de entre 100.000 y 250.000 habitantes, ciudades grandes, de entre 250.000 a 1.000 .000 ;

- Lima Metropolitana, la cual por su magnitud se analiza como un caso particular. La agrupación de las ciudades se observa en la Tabla 1.

La Tabla 2 muestra que, de acuerdo con la categorización, 52 de las ciudades son consideradas pequeñas y representan el 9,5\% de la población. Asimismo, 12 y 9 ciudades son consideradas ciudades medianas y grandes, representando el 7,4 y 14,9\% de la población, respectivamente. Lima Metropolitana alberga el 30,9\% de la población nacional.

Para cada tipo de ciudad (excepto Lima Metropolitana) se realizó un análisis cluster usando el método de partición iterativa K-mean. El análisis cluster permite clasificar a un grupo de individuos, en este caso ciudades, en grupos homogéneos o clusters, de tal modo que los individuos pertenecientes a un cluster sean lo más similares posibles y exista la mayor diferencia posible entre clusters. Las tres variables que se utilizaron para clasificar las ciudades fueron las tres variables de interés del estudio:

- el decrecimiento porcentual del tamaño de las familias; 
- el crecimiento porcentual del ingreso per cápita de los hogares;

- el crecimiento porcentual de la oferta de centros educativos privados.

Posteriormente, haciendo uso de la ENAHO para los años 2012 al $2016^{1}$, se realizó la estimación de la probabilidad de que la población en edad escolar (5 a 16 ańos) se encuentre matriculada en un centro educativo de carácter privado. Las estimaciones que se realizaron son de tipo probit y permiten conocer cómo nuestras variables de interés afectan la probabilidad de que un niño se encuentre matriculado en un centro educativo de carácter privado.

\section{Resultados}

En primer lugar, es importante señalar que la matríacula privada en las zonas urbanas de Perú ha crecido en los últimos diez años. Es decir, más allá del tipo de ciudad y su magnitud, desde el año 2004 se ha presentado un crecimiento acentuado en toda el área urbana.

No obstante, para cada uno de los grupos de ciudades según su tamaño poblacional, el análisis cluster reveló dos tipos de ciudades, las que denominamos como tipo A y tipo B. La diferencia entre ambos tipos de ciudades se ha definido en relación con el crecimiento de la matrícula privada, siendo las del primer tipo las que, en promedio, presentaron un mayor crecimiento de esta matrícula.

Como se puede observar en la Tabla 3, las estadísticas obtenidas para cada uno de los grupos de ciudades. Específicamente, se muestran el promedio del crecimiento porcentual de la matricula privada y los valores promedios de las variables en base a las cuales se determinaron los clusters.

En términos generales, se encontró que las ciudades con mayor crecimiento de la matrícula privada (tipo A) son las que presentaron, también, un

Tabla 2

Clasificación de las ciudades según tamaño poblacional.

\begin{tabular}{l|c|c|c}
\hline Tipo de ciudad & Tamańo de población & $\begin{array}{c}\text { Número de } \\
\text { ciudades }\end{array}$ & $\begin{array}{c}\text { \% de la población } \\
\text { nacional }^{\mathbf{1}}\end{array}$ \\
\hline Pequeńa & Menos de 100.000 habitantes & 52 & 9,5 \\
\hline Mediana & Entre 100.000 y 250.000 habitantes & 12 & 7,4 \\
\hline Grande & Entre 250.000 a 1.000 .000 habitantes & 9 & 14,9 \\
\hline Lima Metropolitana & 8.482 .619 habitantes & 1 & 30,9 \\
\hline Total & & 74 & 62,8 \\
\hline
\end{tabular}

${ }^{1}$ Datos de población provenientes del Censo de Población y Vivienda del 2007. 
mayor crecimiento en las variables de ingreso per cápita y número de centros educativos privados, y un mayor decrecimiento del tamaño familiar. Esto se produjo tanto en las ciudades pequeńas, como en las medianas y grandes.

En particular, en las ciudades de tamaño pequeño, las ciudades de tipo A crecieron un $101,62 \%$ en la matrícula privada, un $120,58 \%$ en el ingreso per cápita, un 54,41\% en la oferta educativa, y decrecieron un 19,31\% en el tamaño de sus familias, mientras que las ciudades de tipo B crecieron un $72,07 \%$ en la matrícula privada, un $26,33 \%$ en el ingreso per cápita, un $20,30 \%$ en el número de centros educativos privados y decrecieron un $17,72 \%$ en el tamańo familiar. Este mismo patrón se aprecia en el resto de las ciudades y es confirmado a través del análisis de regresión.

Por ejemplo, en el caso de las ciudades medianas del tipo A se aprecia que el crecimiento del ingreso per cápita fue del $81,98 \%$ frente a un $60,95 \%$ del incremento de las ciudades medianas de tipo B. En esta misma línea, en el caso del crecimiento del número de centros educativos privados, se observa que en las ciudades medianas de tipo A el aumento es bastante más elevado (100,72\%), que las del tipo B que alcanzan tan solo el $17,60 \%$.

Por otro lado, la Tabla 4 muestra los efectos marginales promedios estimados para cada uno de los años. Los efectos marginales indican en cuánto aumenta o disminuye la probabilidad de la matrícula privada ante un aumento marginal en alguna variable explicativa. Los resultados indican que el número de miembros del hogar, el ingreso per cápita del hogar (en miles de soles) y el número de centros educativos tienen efectos marginales estadísticamente significativos sobre la matrícula privada, controlando por el sexo y la edad del niño, el nivel educativo del jefe del hogar expresado como el número de años de educación, y

\section{Tabla 3}

Estadísticas descriptivas según tipo de ciudad.

\begin{tabular}{l|c|c|c|c|c}
\hline Tipo de ciudad & $\begin{array}{c}\text { Número } \\
\text { de } \\
\text { ciudades }\end{array}$ & $\begin{array}{c}\text { Crecimiento } \\
\text { de la } \\
\text { matricula } \\
\text { privada }\end{array}$ & $\begin{array}{c}\text { Decrecimiento } \\
\text { del tamańo de } \\
\text { las familias }\end{array}$ & $\begin{array}{c}\text { Crecimiento } \\
\text { del ingreso } \\
\text { per cápita }\end{array}$ & $\begin{array}{c}\text { Crecimiento } \\
\text { del número } \\
\text { de centros } \\
\text { educativos } \\
\text { privados }\end{array}$ \\
\hline Ciudad pequeńa (tipo A) & 32 & 101,62 & 19,31 & 120,58 & 54,41 \\
\hline Ciudad pequeńa (tipo B) & 20 & 72,07 & 17,72 & 26,33 & 20,30 \\
\hline Ciudad mediana (tipo A) & 3 & 132,16 & 16,32 & 81,98 & 100,72 \\
\hline Ciudad mediana (tipo B) & 9 & 106,28 & 16,00 & 60,95 & 17,60 \\
\hline Ciudad grande (tipo A) & 6 & 72,29 & 14,86 & 42,03 & 13,60 \\
\hline Ciudad grande (tipo B) & 3 & 49,89 & 14,87 & 99,08 & 7,77 \\
\hline Lima Metropolitana & 1 & 66,98 & 19,01 & 42,80 & $-11,41$ \\
\hline
\end{tabular}


el tipo de área de residencia (rural/urbano). Es decir, se presenta una correlación entre las tres variables de interés, lo que es demostrado en el análisis de regresión. Cabe mencionar que la única variable que pierde significancia es la de tamańo de la familia, pero solo para el último año.

Para el año 2016, en lo que respecta a las variables explicativas de interés, se tiene que un aumento de S/.1000 en el ingreso per cápita aumenta la probabilidad de matrícula privada en 7,4 puntos porcentuales. Asimismo, que haya un centro educativo privado más en el municipio aumenta la probabilidad de matrícula privada en 0,01 puntos porcentuales. Estos resultados no difieren mucho respecto a los resultados que se obtienen para los años anteriores 2012-2015, lo cual muestra la estabilidad del modelo. Por último, se encontró que el aumento de un miembro más en el hogar reduce la probabilidad de estudiar en una institución privada en promedio 0,4 puntos porcentuales durante los años 2012-2015, mientras que para el año 2016 el efecto marginal no fue estadísticamente significativo.

Tabla 4

Efectos marginales promedio estimados.

\begin{tabular}{|c|c|c|c|c|c|}
\hline \multicolumn{6}{|c|}{ Variable dependiente: Matrícula privada } \\
\hline Variables & $\begin{array}{l}\text { Año } \\
2012\end{array}$ & $\begin{array}{c}\text { Año } \\
2013\end{array}$ & $\begin{array}{l}\text { Año } \\
2014\end{array}$ & $\begin{array}{c}\text { Año } \\
2015\end{array}$ & $\begin{array}{c}\text { Año } \\
2016\end{array}$ \\
\hline \multirow{2}{*}{$\begin{array}{l}\text { Total de miembros del } \\
\text { hogar }\end{array}$} & $-0,0034$ & $-0,0051$ & $-0,0031$ & $-0,0056$ & $-0,0018$ \\
\hline & $(0,0010)^{* *}$ & $(0,0010)^{* *}$ & $(0,0010)^{* *}$ & $(0,0010)^{* *}$ & $(0,0010)$ \\
\hline \multirow{2}{*}{$\begin{array}{l}\text { Ingreso per cápita del } \\
\text { hogar }\end{array}$} & 0,0694 & 0,0714 & 0,0872 & 0,0597 & 0,0736 \\
\hline & $(0,0026)^{* *}$ & $(0,0026)^{* *}$ & $(0,0028)^{* *}$ & $(0,0023)^{* *}$ & $(0,0024)^{* *}$ \\
\hline \multirow{2}{*}{$\begin{array}{l}\text { Número de centros } \\
\text { educativos }\end{array}$} & 0,0001 & 0,0001 & 0,0001 & 0,0001 & 0,0001 \\
\hline & $(0,0000)^{* *}$ & $(0,0000)^{* *}$ & $(0,0000)^{* *}$ & $(0,0000)^{* *}$ & $(0,0000)^{* *}$ \\
\hline \multicolumn{6}{|l|}{ Controles: } \\
\hline \multirow{2}{*}{$\begin{array}{l}\text { Dummy si el niño es } \\
\text { mujer }\end{array}$} & $-0,0035$ & $-0,0043$ & $-0,0058$ & $-0,0045$ & $-0,0034$ \\
\hline & $(0,0038)$ & $(0,0035)$ & $(0,0035)$ & $(0,0034)$ & $(0,0034)$ \\
\hline \multirow{2}{*}{ Edad del niño en años } & $-0,0000$ & $-0,0018$ & $-0,0022$ & $-0,0016$ & $-0,0015$ \\
\hline & $(0,0005)$ & $(0,0005)^{* *}$ & $(0,0005)^{* *}$ & $(0,0005)^{* *}$ & $(0,0005)^{* *}$ \\
\hline \multirow{2}{*}{$\begin{array}{l}\text { Años de educación del jefe } \\
\text { de hogar }\end{array}$} & 0,0076 & 0,0083 & 0,0070 & 0,0094 & 0,0080 \\
\hline & $(0,0005)^{* *}$ & $(0,0004)^{* *}$ & $(0,0004)^{* *}$ & $(0,0004)^{* *}$ & $(0,0004)^{* *}$ \\
\hline \multirow{2}{*}{ Dummy de área rural } & $-0,1303$ & $-0,1283$ & $-0,1265$ & $-0,1403$ & $-0,1436$ \\
\hline & $(0,0057)^{* *}$ & $(0,0053)^{* *}$ & $(0,0054)^{* *}$ & $(0,0054)^{* *}$ & $(0,0054)^{* *}$ \\
\hline Observaciones & 24,050 & 27,923 & 27,337 & 28,211 & 30,594 \\
\hline Pseudo $\mathrm{R}^{2}$ & 0,241 & 0,249 & 0,256 & 0,258 & 0,248 \\
\hline
\end{tabular}

Errores estándares entre paréntesis. ${ }^{*} p<0,1 ;{ }^{* *} p<0,05 ;{ }^{* * *} p<0,01$. 
Respecto a las variables de control que se muestran en la Tabla 3, se pueden señalar cuatro principales cuestiones. En primer lugar, se observa que la variable dummy del sexo no es significativa en el caso de la matricula privada. En segundo lugar, indica que a mayor edad del niño la probabilidad de matricularse en una escuela privada es menor. En tercer lugar, se muestra que a más años de educación del jefe de hogar, la probabilidad de que sus hijos sean matriculados en una escuela privada aumentará. Por último, y como se ha señalado al inicio de este apartado, el fenómeno de la expansión de la matrícula privada es básicamente urbano. Como se observa en la Tabla 3, residir en un área rural disminuye la probabilidad de matrícula en una escuela privada en 14.3 puntos porcentuales.

\section{Conclusiones}

El objetivo de este artículo fue presentar el crecimiento de la matrícula escolar en las zonas urbanas de Perú, a partir del comportamiento de tres variables: el incremento de la oferta educativa, el crecimiento económico de las familias y la disminución del tamaño de las familias.

En primer lugar, el estudio muestra que, aunque en distinta medida la matrícula privada ha venido incrementándose durante el período de análisis (2004-2016) en todas las zonas urbanas del país, independientemente de su tamaño poblacional. Este incremento de la matrícula es más evidente en las ciudades pequeñas y medianas, quienes fueron, en muchos casos, impactadas positivamente por los años del boom económico nacional. Efectivamente, entre el 2004 y el 2016 la pobreza urbana se redujo en aproximadamente 35 puntos porcentuales, de acuerdo con las cifras del Sistema de Consulta de Series Nacionales del INEI (2017).

Para todos los casos, el incremento de la matrícula privada ha venido acompañado de un incremento en el ingreso per cápita, en la oferta de escuelas privadas y en la disminución del tamaño de las familias, salvo el caso de Lima Metropolitana en donde la oferta decreció. Este decrecimiento de la oferta en Lima no supone una disminución de la matrícula, sino que los colegios se han hecho "más grandes" y han surgido "cadenas" de escuelas provenientes de consorcios educativos (ROMÁN \& RAMÍREZ, 2018).

El fenómeno de expansión de la matrícula privada ha dejado de ser un asunto propio de Lima para convertirse en un proceso nacional urbano. Aun cuando en Lima Metropolitana el crecimiento de la matrícula privada es exponencial, en tanto concentra el $49 \%$ de la matrícula de la ciudad y un tercio de la matrícula privada del país (BALARÍN et al. 2018), el crecimiento en el resto urbano del país viene sostenidamente creciendo. 
Finalmente, el número de miembros del hogar, el ingreso per cápita del hogar y el número de centros educativos tienen efectos estadísticamente significativos sobre la matrícula privada. El ingreso per cápita y la existencia de oferta privada son las variables que más explican el crecimiento de la matrícula privada.

Como se ha señalado en diversos estudios (CUENCA, 2013, BALARÍN, 2016), este incremento de la oferta privada no ha supuesto, necesariamente, una mejora en la calidad de las escuelas. Por el contrario, la escuela privada que se ha expandido en Perú es la de bajo costo y, en muchas ocasiones, de menor calidad.

No obstante, si bien a través de este análisis no se puede dar cuenta de la calidad del tipo de oferta a la que acceden estas nuevas familias, se podría indicar que aún existe una aspiración por matricular a sus hijos en escuelas privadas (ROMÁN \& RAMÍREZ, 2018; SANZ, 2014). La mejora de los ingresos familiares y la reducción del número de hijos, ha conllevado a que las familias decidan matricular a sus hijos en una institución de este tipo, dejando de lado la opción de la escuela pública.

Cabe mencionar, en esta línea, que las variables de control como los años de educación del jefe de hogar y la edad del niño influyen en la matricula privada. Es decir, si los padres tienen más años de educación accederán a matricular a sus hijos a la educación privada, frente a la educación pública. Así también, los niños más pequeños también acceden más a la oferta privada, lo que podría resultar en un cambio generacional en el acceso a la matricula.

Si bien la educación privada generaría externalidades positivas como las network externalities, la masificación de la oferta privada reproduciría desigualdades sociales (GALLEGO, 2004) y se asociaría a mayores niveles de segregación tanto de los estudiantes como de los resultados (AUGUSTE \& VALENZUELA, 2004); particularmente, por niveles socioeconómicos (MURILLO; BELAVI; PINILLA, 2018) y por factores socioculturales (ELACQUA et al., 2012). Uno de los principales retos de los sistemas educativos y de los Estados es reconfirmar que la educación es un bien público que beneficia más a la sociedad, que al individuo particular. Por esto y otras razones, las fallas de mercado son tan evidentes en la educación. En suma, resulta fundamental acompańar la expansión de la iniciativa privada en educación con estrategias adecuadas de regulación de la calidad, y la promoción transparente de información y claridad de los fines públicos y sociales de la educación (ROBERTSON \& VERGER, 2012), pues con inequidades de servicio educativo, segregaciones educativas y la desnaturalización de la función social de la educación no es posible ni desarrollo, ni democracia. 
Nota

1. Antes del año 2012 no se cuentan los datos del tipo de institución (privada o pública) a la que se encuentran matriculados todos los individuos, sino únicamente se tiene este dato para aquellos individuos que, al momento de la encuesta, asistían a clases.

\section{Referencias}

AUGUSTE, S.; VALENZUELA, J.P. Do Students Benefit from School Competition? The Chilean Experiencia. Tesis (Grado) - University of Michigan, Michigan, 2004.

BAGLEY, C.; WOODS, P.; GLATTER, R. School Choice and Competition: Markets in the Public Interest? Londres: Routledge, 2005.

BALARÍN, M. La privatización por defecto y el surgimiento de las escuelas privada. RASE, v. 9, n. 2, p. 181-196, 2016.

BALARÍN, M. The Default Privatization of Peruvian Education and the Rise of Lowfee Private Schools: Better or Worse Opportunities for the Poor? ESP Working Papers, n. 65, 2015.

BALARÍN, M.; KITMANG, J.; ÑOPO, H.; RODRIGUEZ, M. Mercado privado, consecuencias públicas: los servicios educativos de provisión privada en el Perú. Lima: GRADE, 2018. (Documento de investigación, n. 89).

BELLEI, C. Expansión de la educación privada y mejoramiento de la educación en Chile. Evaluación a partir de la evidencia. Pensamiento Educativo, v. 40, n. 1, p. 285$311,2007$.

BELLO, M.; VILLARÁN, V. Educación, reformas y equidad en los países de los andes y cono sur: dos escenarios en el Perú. Buenos Aires: IIPE-UNESCO, 2004.

BENAVIDES, M.; LEON, J.; HAAG, F.; CUEVA, S. Expansión y diversificación de la educación superior universitaria, y su relación con la desigualdad y la segregación. Lima: GRADE, 2015. (Documento de investigación, n. 78).

BENAVIDES, M. Lejos (aún) de la equidad: la persistencia de las desigualdades educativas en el Perú. In: GRUPO DE ANÁLISIS PARA EL DESARROLLO (org.). Investigación, políticas y desarrollo en el Perú. Lima: Grade, 2007.

BENAVIDES, M.; LEÓN, J.; ETESSE, M. Desigualdades educativas y segregación en el sistema educativo peruano: una mirada comparativa de las pruebas PISA 2000 y 2009. Lima: GRADE, 2014. (Avances de Investigación, n. 15).

CADDELL, M.; ASHLEY, L.D. Blurring boundaries: towards a reconceptualisation of the private sector in education. Compare, v. 36, n. 4, p. 411-419, 2006. https://doi. org/10.1080/03057920601024750

CASTRO, J.; YAMADA, G. Brechas étnicas y de sexo en el acceso a la educación básica y superior en el Perú. Perú, 2011. (Documento de Discusión, n. 11/04). 
CUENCA, R. Desencuentros entre el discurso del derecho a la educación y las políticas educativas en el Perú de los 2000. Lima: Instituto de Estudios Peruanos y Consejo Latinoamericano de Ciencias Sociales, 2012. (Documento de Trabajo, 170. Serie Educación, 4).

CUENCA, R. La escuela pública en Lima Metropolitana. ¿Una institución en extinción? Revista Peruana de Investigación Educativa, n. 5, p. 73-98, 2013.

CUENCA, R. Reconfiguraciones entre Estado y ciudadanía. Imaginarios de éxito y nuevas políticas sociales. In: GROMPONE, R. (org.). Incertidumbres y distancias: el controvertido protagonismo del Estado en el Perú. Lima: Instituto de Estudios Peruanos, 2016. p. 447-478.

CUENCA, R.; REÁTEGUI, L. La (incumplida) promesa universitaria. Lima: IEP, 2016. (Documento de Trabajo, 230. Serie Educación, 11).

CUENCA, R.; PATIÑO, P. Profesionalización femenina desde los márgenes. Trayectorias de mujeres indígenas hacia la educación superior. In: VARGAS, S. (org.). Bajo el radar de Sofía: Oportunidades y barreras de las profesionales en el Perú. Lima: IEP, 2014.

DE BELAUNDE, C. Profundizando las brechas: una mirada a la desigualdad en los estudios sobre el sistema educativo peruano. In: COTLER, J.; CUENCA, R. (orgs.). Las desigualdades en el Perú: balances críticos. Lima: IEP, 2011. p. 273-329.

DÍAZ, H.; HUAYTE, V.; FARRO, F.; TÁVARA, J. La educación privada en Lima Metropolitana. Buenos Aires: Instituto Internacional de Planeamiento de la Educación de la Unesco, 1995. (Informe de Investigación del IIPE, n. 104).

DU BOIS, F.; CHÁVEZ, J.; CUSANTO, A. Programas sociales, salud y educación en el Perú: un balance de las políticas sociales. Lima: Instituto Peruano de Economía Social de Mercado y Fundación Konrad Adenauer, 2004.

ELACQUA, G.; MARTÍNEZ, M.; SANTOS, H.; URBINA, D. Tensiones públicoprivado y el diseño de los sistemas educativos: ¿Qué nos dice PISA? Pro-Posiçóes, v. 23, n. 2 (68), p. 105-123, 2012.

GALLEGO, F. School Choice, Incentives, and Academic Outcomes: Evidence from Chile.

Research Papers in Economics, 2014. Disponible en: shttp://repec.org/esLATM04/ up.17368.1080314323.pdf>. Acceso el: 22 nov. 2017.

GUADALUPE, C.; LEON, J.; RODRIGUEZ, J.; VARGAS, S. Estado de la educación en el Perú: análisis y perspectivas de la educación básica. Lima: FORGE-GRADE, 2017.

INSTITUTO NACIONAL DE ESTADÍSTICA E INFORMÁTICA (INEI). Perú: Estimaciones y proyecciones de población total por sexo de las principales ciudades, 20122015. Lima: INEI. Disponible en: <https://www.inei.gob.pe/media/MenuRecursivo/ indices tematicos/cap03027.xls $>$. Acceso en el: 30 set. 2017.

INSTITUTO NACIONAL DE ESTADÍSTICA E INFORMÁTICA (INEI). Series nacionales. Lima: INEI, 2017. Disponible en: <http://webapp.inei.gob.pe:8080/sirtod-

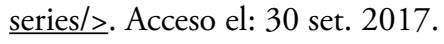


IREGUI, A.M.; MELO, L.; RAMOS, J. Análisis de eficiencia de la educación en Colombia. Revista Económica del Rosario, v. 10, n. 1, p. 21-41, 2007.

LAMAS, L. La universidad privada en la construcción de subjetividades juveniles emprendedoras. Revista Peruana de Investigación Educativa, n. 7, p. 127-151, 2015.

LARRAÑAGA, O. Competencia y participación privada: la experiencia chilena en educación. Santiago de Chile: Universidad de Chile, 2004.

MIRANDA, L. Factores asociados al rendimiento escolar y sus implicancias para la política educativa del Perú. I: BENAVIDES, M. (org.). Análisis de programas, procesos y resultados educativos en el Perú: contribuciones empíricas para el debate. Lima: GRADE, 2008.

MURILLO, F.J. Midiendo la segregación escolar en América Latina. Un análisis metodológico utilizando el TERCE. REICE. Revista Iberoamericana sobre Calidad, Eficacia y Cambio en Educación, v. 14, n. 4, p. 33-60, 2016. https://doi.org/10.15366/ reice2016.14.4.002

MURILLO, J.; BELAVI, G.; PINILLA, L. Segregación escolar público-privada en España. Papers. Revista de Sociología, v. 103, n. 3, p. 307-337, 2018. https://doi.org/10.5565/ rev/papers.2392

NÚNEEZ, J.; STEINER, R.; CADENA, X.; PARDO, R. ¿Cuáles colegios ofrecen mejor educación en Colombia? Bogotá: Dirección de Estudios Económicos del Departamento Nacional de Planeación, 2002.

ORTIZ DE ZEVALLOS, G.; EYZAGUIRRE, H.; PALACIOS, R.; POLLAROLO, P. La economía política de las reformas institucionales en el Perú: los casos de educación, salud y pensiones. Lima: Banco Interamericano de Desarrollo, 1999.

RIVAS, A. América Latina después de PISA: lecciones aprendidas de la educación en siete países 2000-2015. Buenos Aires: CIPPEC-Natura, 2015.

ROBERTSON, S.; MUNDY, K.; VERGER, A.; MENASHY, F. An introduction to public private partnerships and educaction governance. In: ROBERTSON, S.; MUNDY, K.; VERGER, A.; MESASHY, F. (orgs.). Private public partnerships in education. New actors and modes of governance in a globalizing world. Massachusetts: Edward Elgar, 2012.

ROBERTSON, S.; VERGER, A. Governing education through public private partnerships. In: ROBERTSON, S.; MUNDY, K.; VERGER, A.; MESASHY, F. (orgs.). Private public partnerships in education. New actors and modes of governance in a globalizing world. Massachusetts: Edward Elgar Publishing, 2012.

ROMÁN, A.; RAMÍREZ, A. ¿El mito sigue vivo? Privatización y diferenciación social en la educación peruana. Lima: IEP, 2018.

SANZ, P. "We don't need the state". A study of the habitus formation process, through school choice, in the Peru's rising middle class. A qualitative study of school choice. Tesis (Doctorado) - Universidad de Bath, 2014. 
SANZ, P. El tránsito de la escuela pública a la escuela privada en el sector emergente de Lima Metropolitana: ¿Buscando mejor calidad? Revista Peruana de Investigación Educativa, n. 7, p. 95-125, 2015.

SPARROW, B.; PONCE DE LEÓN, M. Gaps in cognitive and socio-emotional development between public and private school children in Peru's urban areas. Working ESP-WP, N 70. Lima: Privatisation in Education Research Iniciative, 2015.

\section{SOBRE LOS AUTORES}

Ricardo Cuenca es doctor en Educación. Investigador principal del Instituto de Estudios Peruanos.

Luciana Reátegui Amat y León es licenciada en Sociología. Investigadora del Instituto de Estudios Peruanos.

SARIta OrÉ es magister en Economía. Analista senior del Ministerio de Economía y Finanzas del Perú.

Recibido el 3 de marzo de 2018.

Aceptado el 6 de marzo de 2019. 